\title{
Thermoelastic analysis of FGM hollow cylinder for variable parameters and temperature distributions using FEM
}

https://doi.org/10.1515/nleng-2020-0013

Received Feb 5, 2019; accepted Feb 5, 2020.

\begin{abstract}
This paper presents, numerical study of stress field in functionally graded material (FGM) hollow cylinder by using finite element method (FEM). The FGM cylinder is subjected to internal pressure and uniform heat generation. Thermoelastic material properties of FGM cylinder are assumed to vary along radius of cylinder as an exponential function of radius. The governing differential equation is solved numerically by FEM for isotropic and anistropic hollow cylinder. Additionally, the effect of material gradient index $(\beta)$ on normalized radial stresses, normalized circumferential stress and normalized axial stress are evaluated and shown graphically. The behaviour of stress versus normalized radius of cylinder is plotted for different values of Poisson's ratio and temperature. The graphical results shown that stress field in FGM cylinder is influenced by some of above mentioned parameters.
\end{abstract}

Keywords: FGM, cylinder, FEM, thermo-mechanical parameters

\section{Introduction}

Functionally graded materials have great application in fields of aerospace, automobiles, industry, defence, energy, electronics, electrical, biomedical and sports. FGMs are used widely due to variation in composition and structural gradually over volume that results in variation in chemical, thermal, electrical and mechanical properties. These materials can be constructed for some special application and function. In FGMs material properties are not homogeneous across the whole material. FGM cylin-

\footnotetext{
*Corresponding Author: Dinkar Sharma, Department of Mathematics, Lyallpur Khalsa College, Jalandhar-144001, Punjab, India; E-mail: dinkar.nitj@gmail.com

Ramandeep Kaur, Department of Mathematics, I.K.G. Punjab Technical University, Jalandhar-144603, Punjab, India
}

drical structures have number of applications in field of engineering and science, therefore number of theoretical and experimental studies have been done to optimize the weight, mechanical strength, displacement, stress and strain of cylinder. In past decade of years, researchers and scientists made analysis of different types of cylinders.

Dai et al. [1] presented exact solution for stresses in functionally graded metal hollow cylinder by using infinitesimal theory of magneto-thermoelasticity. Abrinia et al. [2] obtained radial and circumferential stress in FGM cylindrical vessel under internal pressure and temperature from analytical solution. Further, effect of non homogeneity in FGM thick cylinder was discussed by choosing dimensionless parameters. Evci and Gulgec [3] developed analytical solution to present stress and displacement in hollow cylinder under heat generation and internal pressure that analytical solution derived with help airy stress function. Rahimi and Nejad [4] used theory of elasticity to find exact solution in hollow thick walled rotating FGM cylinder under internal and external pressure. Sharma et al. [5] made analysis of stress and strain with help of finite element in rotating circular disk under exponentially varing material properties. Sharma et al. [6] studied effect of Kibel number on thermoelastic characteristics in FGM disk by using FEM. Naghdabadi and Kordkheii [7] taken power law distribution model of material properties to made thermoelastic analysis of functionally graded plates and shells by FEM. Go [8] analyzed thermoelastic characteristics of circular disk under effect of rotating speed and radial thickness by using FEM. FEM was used by number of researchers to study numerical behaviour of thermoelastic characteristics. The detailed literature related to FEM is given in [9-12]. Yadav and Jiwari [13-15] used FEM to solve different types of problem related to differential equations i.e. FEM used solve Burger's Fisher's equation, srusselator model and coupled reaction diffusion model. Sharma et al. [16] investigated thermoelastic characteristics in FGM disk under linearly varing material properties by using FEM. Sharma and Kaur [17] made comparison of stress and strain for two FGM disks where first disk constructed from Aluminum and Alumina and second 
disk constructed from Zirconium and Zirconium oxide. To carried out comparison of different thermoelastic characteristics FEM was used. Farhan et al. [18] used finite difference method to problem of thermoelasticity for an infinitely long and isotropic circular cylinder. This method presented numerical solution for displacement, temperature and stress under mechanical and thermal boundary conditions. Habib et al. [19] developed mathematical analysis to study stresses and strains in FGM cylinder under exponentially varing material properties. Also FEM and ANSYS software had been used to compute different values of stress. Vaziri et al. [20] described analytical formulation for FGM thick walled cylinder with power law variation in properties. Further, FEM was used to checked validation of analytical solution. Bayat et al. [21] made analysis of a rotating functionally graded disk under two types of thickness profiles and steady temperature field. Liew et al. [22] used novel limiting process to study thermal stress and temperature in hollow circular cylinder. Jabbari et al. [23] developed analysis of short length hollow FGM cylinder with help of generalized bessel function. This analysis was carried out under radial and longitudinal direction of temperature. Parveen et al. [24] used FEM to find two step solution of governing differential equation of thermoelasticity in ceramic-metal cylinder. Jabbari et al. [25] studied thermal stresses in FGM cylinder, in this cylinder temperature distribution and other material properties were assumed to vary as function of radius. Nejad et al. [26] presented elastic analysis of FGM thickwalled cylindrical pressure vessels from its analytical solution under exponential varing material properties. Eslami et al. [27] investigated analytical solution for functionally graded hollow thick sphere under general thermal mechanical boundary conditions and radially varing material properties. Peng and Lie [28] converted thermoelastic problem to fredholm integral equation for obtaining thermal stress in functionally graded hollow cylinder. Yeo et al. [29] used recursive method to find analytical solution in hollow multilayered cylinder. Further, from this analytical solution stress/displacement was found under thermomechanical loading. Atli and Lak [30] presented analysis of functionally graded piezoelectric hollow cylinder by obtaining stresses, strains and displacements under action of internal and external pressure and temperature gradinent. Tutuncu [31] studied stresses and displacements in functionally graded cylindrical vessels from power series solution under constant Poisson's ratio and exponentially varing elastic modulus. Wang [32] made transient thermal analysis in functionally graded hollow cylinder under heat conductivity, mass density and specific heat which all were vary along radial direction. Tanvir et al. [33] de- rived stress and strain in FGM cylinder under the effect of internal pressure, temperature difference, thickness and material distribution. Zheng et al. [34] used finite difference method to obtain stress in functionally graded rotating disk, where elastic modulus and mass density follows power law function of radius of disk. Zenkour [35] described effects of temperature and moisture concentration on piezoelectric cylinder, that cylinder subjected to external pressure and electronic potential. Mathena et al. [36] carried out heat conduction and thermal stress in hollow cylinder with non homogeneous material properties. Saddalfar [37] analyzed stress distribution in piezomagnetic rotating thick walled cylinder from constitutive equations.

This paper Investigates, stresses in FGM hollow cylinder subjected to internal pressure and uniform heat generation. Thermoelastic material properties such as thermal expansion coefficient, modulus of elasticity, thermal conductivity and yield stress are taken as exponential function of radius of cylinder. By using equilibrium equation in cylinder and Hooke's law problem is transform to second order differential equation. Finite element method is used to find numerical solution of differential equation for isotropic and anisotropic hollow cylinder. Furthermore, effect of Poisson's ratio and temperature on normalized radial, circumferential and axial stress represented graphically. The analysis shows that in FGM hollow cylinder stresses can be reduced by taking particular values of material parameters.

\section{Modelling of cylinder}

A FGM cylinder with inner and outer radius $a$ and $b$, respectively is considered, while $T_{i}$ and $T_{0}$ are the temperatures at inner and outer surfaces of cylinder. The material properties named as coefficients of thermal expansion, modulus of elasticity, coefficient of thermal conductivity and yield stress of cylinder are modelled by exponential variation as shown below:

$$
\begin{gathered}
\alpha(r)=\alpha_{0} r^{\beta_{1}} \\
E(r)=E_{0} r^{\beta_{2}} \\
\lambda(r)=\lambda_{0} r^{\beta_{3}} \\
\sigma_{y}(r)=\sigma_{0} r^{\beta_{4}}
\end{gathered}
$$

Where $\alpha_{0}, E_{0}, \lambda_{0}, \sigma_{0}$ are material constants and $\beta_{1}, \beta_{2}, \beta_{3}, \beta_{4}$ are power law exponents corresponding 
to thermal expansion coefficient, modulus of elasticity, thermal conductivity coefficient and yield stress, respectively.

\section{Basic equations}

We consider general form of heat diffusion equation and boundary conditions in homogenous cylinder given by [3] as:

$$
\begin{gathered}
\frac{1}{r} \frac{\partial}{\partial r}\left(\lambda r \frac{\partial T}{\partial r}\right)+\frac{1}{r^{2}} \frac{\partial}{\partial \theta}\left(\lambda \frac{\partial T}{\partial \theta}\right)+\frac{\partial}{\partial Z}\left(\lambda \frac{\partial T}{\partial Z}\right)+q=\rho C_{p} \frac{\partial T}{\partial t} \\
\frac{d T}{d r}=0, \text { at } r=a \text { and } T(r)=T_{0} \text { at } r=b
\end{gathered}
$$

Where $r, \theta, z$ are cylindrical coordinates, $t$ is time, $T$ is temperature distribution, $q$ is heat generation per unit time and per unit volume, $\rho$ is density, $C_{p}$ is specific heat.

Since cylinder is constructed with FGM material therefore, problem is assumed to follow axisymmetric temperature distribution. The heat equation (5) reduces to steady state heat equation as:

$$
\frac{1}{r} \frac{d}{d r}\left(\lambda r \frac{d T}{d r}\right)+q=0
$$

After substituting the value of thermal conductivity in equation (6), we obtained temperature distribution as written below:

$$
T(x)=\frac{-q}{2 \lambda_{0}\left(2-\beta_{3}\right)} r^{2-\beta_{3}}-C_{1 t} r^{-\beta_{3}}+C_{2 t} .
$$

By applying two boundary conditions, we find the values of constants $\left(C_{1 t}, C_{2 t}\right)$ and temperature distribution takes form as given below:

$$
\begin{aligned}
T(r)= & \frac{-q}{2 \lambda_{0}\left(2-\beta_{3}\right)} r^{2-\beta_{3}}-\frac{a^{2} q}{2 \lambda_{0} \beta_{3}} r^{-\beta_{3}}+T_{0}+\frac{q b^{2-\beta_{3}}}{2 \lambda_{0}\left(2-\beta_{3}\right)} \\
& +\frac{a^{2} q b^{-\beta_{3}}}{2 \lambda_{0} \beta_{3}} .
\end{aligned}
$$

\section{Problem formulation}

With help of equilibrium equation in cylinder, we formulate the problem as follows:

$$
\frac{d \sigma_{r}}{d r}+\frac{\sigma_{r}-\sigma_{\theta}}{r}=0 .
$$

Where $\sigma_{r}$ denotes radial stress component, $\sigma_{\theta}$ indicates circumferential stress component and $r$ denotes radial coordinate of cylinder. By using Hooke's law, relationship between stress and strain components is written as:

$$
\epsilon_{r}=\frac{1}{E}\left[\sigma_{r}-v\left(\sigma_{\theta}+\sigma_{z}\right)\right]+\alpha T
$$

$$
\begin{gathered}
\epsilon_{\theta}=\frac{1}{E}\left[\sigma_{\theta}-v\left(\sigma_{r}+\sigma_{z}\right)\right]+\alpha T \\
\epsilon_{z}=\frac{1}{E}\left[\sigma_{z}-v\left(\sigma_{\theta}+\sigma_{r}\right)\right]+\alpha T
\end{gathered}
$$

Where $\epsilon_{r}, \epsilon_{\theta}$ and $\epsilon_{z}$ denotes radial, circumferential and axial components of strain. The axial strain is independent of radial coordinate for FGM cylinder with fixed ends. Therefore its value is taken as constant.

$$
\epsilon_{z}=\frac{1}{E}\left[\sigma_{z}-v\left(\sigma_{\theta}+\sigma_{r}\right)\right]+\alpha T=\epsilon_{0}
$$

From equation (13) axial stress component can be written as:

$$
\sigma_{z}=v\left(\sigma_{r}+\sigma_{\theta}\right)+\left(\epsilon_{0}-\alpha T\right) E
$$

After putting value of axial stress component in equations (11) and (12), these equations can takes the form as written below:

$$
\epsilon_{r}=\frac{1}{E}\left[\left(1-v^{2}\right) \sigma_{r}-v(1+v) \sigma_{\theta}+(1+v) E \alpha T-v \epsilon_{0} E\right]
$$

$$
\epsilon_{\theta}=\frac{1}{E}\left[\left(1-v^{2}\right) \sigma_{\theta}-v(1+v) \sigma_{r}+(1+v) E \alpha T-v \epsilon_{0} E\right]
$$

The equilibrium equation in terms of stress function can be written as:

$$
\sigma_{r}=\frac{\phi}{r}, \quad \sigma_{\theta}=\frac{d \phi}{d r}
$$

The radial and circumferential strain components are related to radial coordinates as written below:

$$
\begin{gathered}
\epsilon_{r}=\frac{d u}{d r} \\
\epsilon_{\theta}=\frac{u}{r}
\end{gathered}
$$

By using relation between radial and circumferential strain components as given in (18) and (19), we obtain a differential equation as given below:

$$
\frac{\epsilon_{r}-\epsilon_{\theta}}{r}-\frac{d \epsilon_{\theta}}{d r}=0
$$

With help of equation (20), we find differential equation of problem as written below:

$$
\begin{aligned}
& r^{2} \frac{d^{2} \phi}{d r^{2}}+\left(1-\beta_{2}\right) r \frac{d \phi}{d r}-\left(1-\frac{v \beta_{2}}{1-v}\right) \phi= \\
& \frac{E_{0} \alpha_{0} q}{2(1-v) \lambda_{0}}\left[\frac{\beta_{1} b^{2-\beta_{3}}}{\beta_{3}-2} r^{\beta_{1}+\beta_{2}+1}+\frac{\beta_{1}-\beta_{3}+2}{2-\beta_{3}} r^{\beta_{1}+\beta_{2}-\beta_{3}+3}\right. \\
& \left.-\frac{a^{2} b^{-\beta_{3}}}{\beta_{3}} r^{\beta_{1}+\beta_{2}+1}+\frac{a^{2}\left(\beta_{1}-\beta_{3}\right)}{\beta_{3}} r^{\beta_{1}+\beta_{2}-\beta_{3}+1}\right]
\end{aligned}
$$


The equation (21) in simplest form can be written as:

$r^{2} \frac{d^{2} \phi}{2 r^{2}}+P_{1} \frac{d \phi}{2 r}-P_{2} \phi=$

$P_{3}\left[C_{1} r^{\beta_{1}+\beta_{2}+1}+C_{2} r^{\beta_{1}+\beta_{2}-\beta_{3}+3}-C_{3} r^{\beta_{1}+\beta_{2}+1}+C_{4} r^{\beta_{1}+\beta_{2}-\beta_{3}+1}\right]$

$\frac{d^{2} \phi}{d r^{2}}+\frac{P_{1}}{r} \frac{d \phi}{d r}-\frac{P_{2}}{r^{2}} \phi=$

$P_{3}\left[C_{1} r^{\beta_{1}+\beta_{2}-1}+C_{2} r^{\beta_{1}+\beta_{2}-\beta_{3}+1}-C_{3} r^{\beta_{1}+\beta_{2}-1}+C_{4} r^{\beta_{1}+\beta_{2}-\beta_{3}-1}\right]$

Where $P_{1}=\left(1-\beta_{2}\right), P_{2}=\left(1-\frac{v \beta_{2}}{1-v}\right), P_{3}=$ $\frac{E_{0} \alpha_{0} q}{2(1-v) \lambda_{0}}, C_{1}=\frac{\beta_{1} b^{2-\beta_{3}}}{\beta_{3}-2}, C_{2}=\frac{\beta_{1}-\beta_{3}+2}{2-\beta_{3}}, C_{3}=\frac{a^{2} b^{-\beta_{3}}}{\beta_{3}}$ and $C_{4}=$ $\frac{a^{2}\left(\beta_{1}-\beta_{3}\right)}{\beta_{3}}$.

\section{Finite element solution of problem}

In this problem, a standard discretization approach of finite element method is used to solve the differential equation (22). In this discretization the size of each element is equal and total domain is divided into $\mathrm{N}$ elements and then equation is converted into simultaneous equations.

$$
\sum_{j=1}^{2} K_{i j}^{e} F_{j}^{e}=L_{i}^{e} ; \quad i=1,2 ; \quad e=1,2,3, \ldots, n
$$

Where the value of $K_{i j}^{e}$ and $L_{i}^{e}$ is obtained from

$$
\begin{gathered}
K_{i j}^{e}=\int_{r_{e}}^{r_{e+1}} \frac{d \varphi_{i}^{e}}{d r} \frac{d \varphi_{j}^{e}}{d r} d r+P_{1} \int_{r_{e}}^{r_{e+1}} \frac{\phi_{i}}{r} \frac{d \phi_{j}}{d r} d r \\
-P_{2} \int_{r_{e}}^{r_{e+1}} \frac{1}{r^{2}} \phi_{i} \phi_{j} d r+\left[\phi_{i} \frac{d \phi_{j}}{d r}\right]_{r_{e}}^{r_{e+1}} \\
L_{i}^{e}=P_{3} \int_{r_{e}}^{r_{e+1}} \varphi_{i}^{e}\left(C_{1} r^{\beta_{1}+\beta_{2}-1}+C_{2} r^{\beta_{1}+\beta_{2}-\beta_{3}+1}-C_{3} r^{\beta_{1}+\beta_{2}-1}\right. \\
\left.+C_{4} r^{\beta_{1}+\beta_{2}-\beta_{3}-1}\right) d r
\end{gathered}
$$

Where

$$
\varphi_{1}^{e}=\frac{r_{e+1}-r}{r_{e+1}-r_{e}}, \varphi_{2}^{3}=\frac{r-r_{e}}{r_{e+1}-r_{e}} .
$$

\section{Application}

To achieve numerical results, we assumed that inner radius $a$ as $1 \mathrm{~mm}$ and outer radius $b$ as $10 \mathrm{~mm}$ respectively.
Dimensionless terms for stresses and radial coordinate are defined as:

Dimensionless stress components: $\bar{\sigma}_{r}=\frac{\sigma_{r}}{\sigma_{0}}, \bar{\sigma}_{\theta}=\frac{\sigma_{\theta}}{\sigma_{0}}, \bar{\sigma}_{z}=$ $\frac{\sigma_{z}}{\sigma_{0}}$

Dimensionless radial coordinate: $R=\frac{r}{b}$

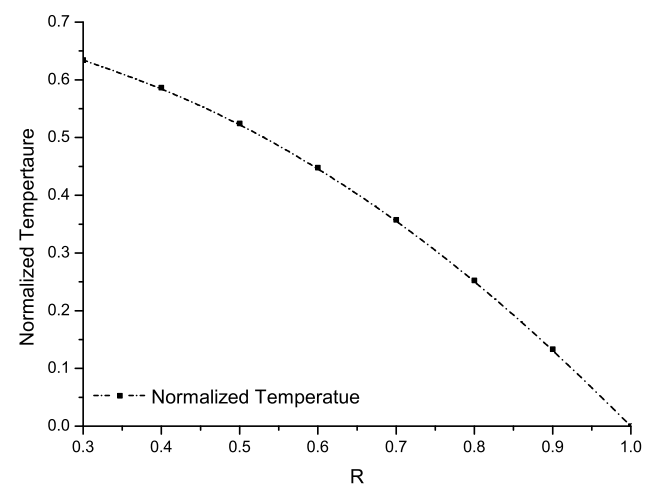

Figure 1: Represents normalized temperature and dimensionless radial coordinate

As shown in Figure 1, the highest temperature achieved at inner surface of cylinder and as moved towards outer surface the value of temperature decreases. The maximum normalized temperature obtained when $R=0.3$ and minimum value for same is attainted at $R=1$. The different cases of stress and displacement are calculated numerically by FEM for FGM hollow cylinder and presented graphically as shown below:

CASE I: For Isotropic hollow cylinder $\left(\beta_{1}=\beta_{2}=\beta_{3}=0\right)$ :

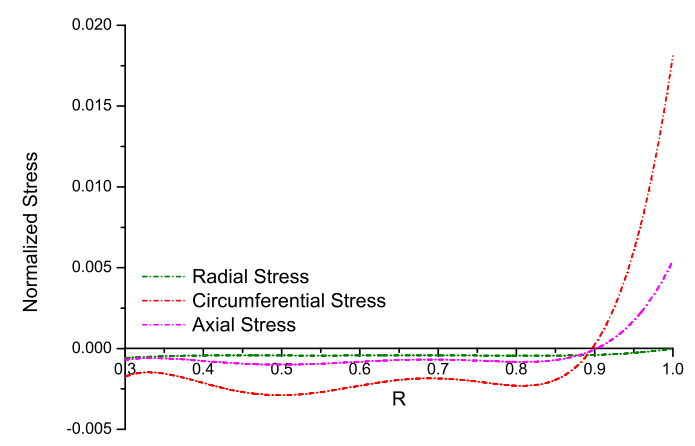

Figure 2: Normalized stresses versus dimensionless radial coordinate 
Figure 2 display normalized stresses against dimensionless radial coordinate for isotropic hollow cylinder. The cylinder is isotropic because values of all material parameters taken as zero $\left(\beta_{1}=\beta_{2}=\beta_{3}=0\right)$. From graph it is cleared that, radial stress is compressive in nature where as circumferential stress and axial stress are compressive in nature at inner surface of cylinder and tensile as moved toward outer surface of cylinder. The maximum value of stresses exists at outer surface of cylinder.

CASE II: Anisotropic hollow cylinder $\left(\beta_{1}=\beta_{2}=\beta_{3}=\beta\right)$ :

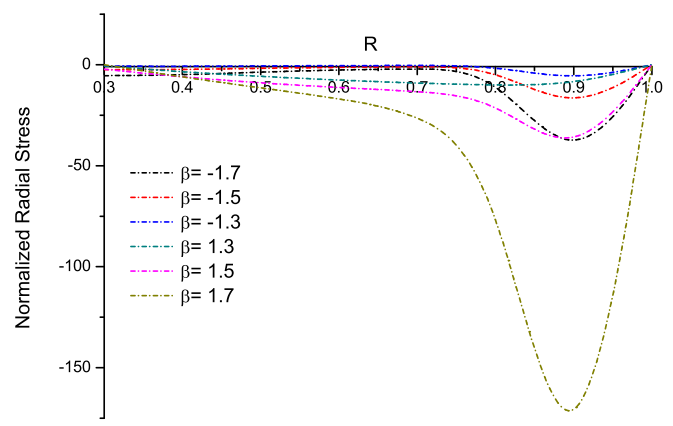

Figure 3: Normalized radial stress and dimensionless radial coordinate for different values of $\beta$

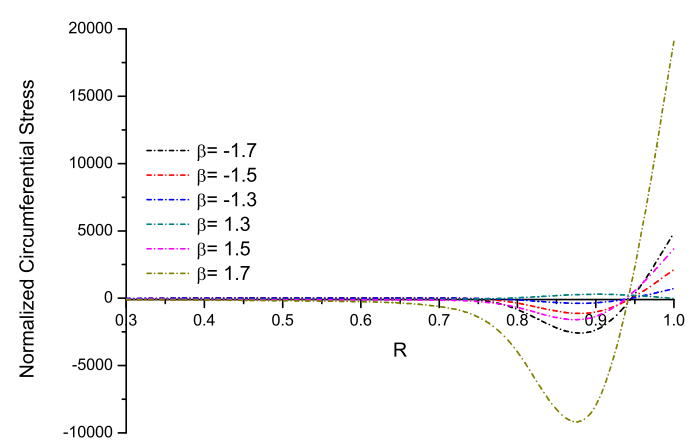

Figure 4: Normalized circumferential stress and radial coordinate for different values of $\beta$

Figure 3-5 represents normalized stresses (normalized radial stress, normalized circumferential stress and normalized axial stress) for different values of material

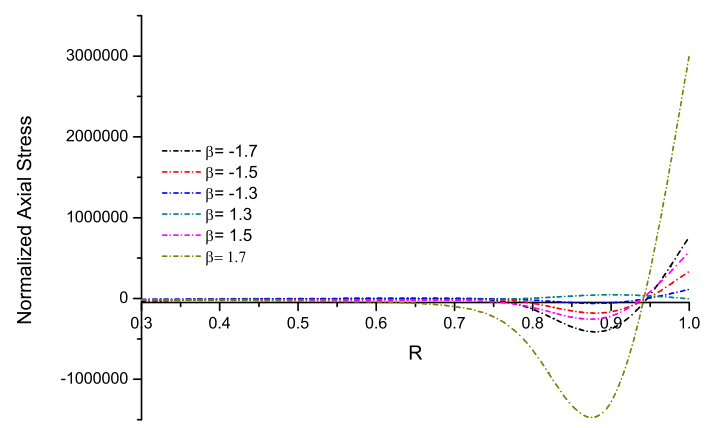

Figure 5: Normalized axial stress and radial coordinate for different values of $\beta$

properties. We take the values of $\beta$ in range from -1.7 to 1.7. As from Figure 3, it is cleared that, value of normalized radial stress is less for higher value of $\beta$ (1.7). Also, normalized radial stress curves are more flexible in nature for positive values of $\beta$. On the other hand the higher value of normalized circumferential stress is obtained for higher values of $\beta$. The normalized circumferential stress is near zero for $\beta=-1.3$ and $\beta=1.3$ and normalized circumferential stress is more variable in nature for $0.7 \leq R \leq 1$. The behaviour of normalized axial stress curves are of same in nature as normalized circumferential stress curves. The axial stress is positive for $\beta=-1.3$ and $\beta=1.3$ but for other values of $\beta$ it changes from positive to negative and than negative to positive.

\section{CASE III: For different values of temperature:}

In this case, thermoelastic characteristics are calculated for FGM hollow cylinder under different values of $T_{0}$.

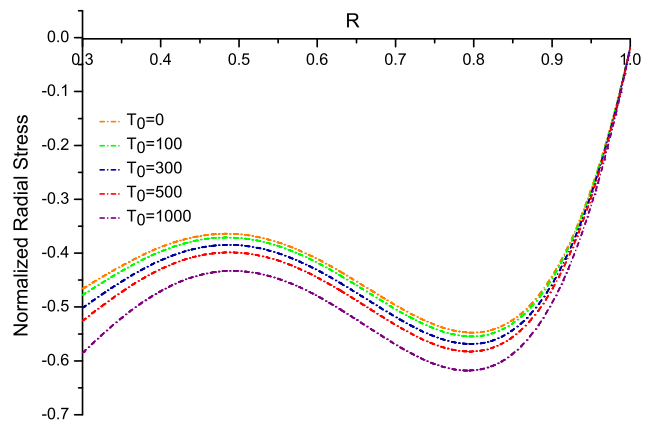

Figure 6: Normalized radial stress distribution in FGM hollow cylinder with different values $T_{0}$ 


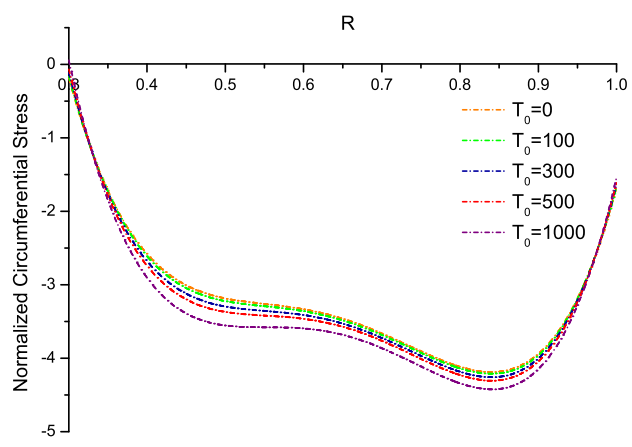

Figure 7: Normalized circumferential stress distribution in FGM hollow cylinder with different values of $T_{0}$

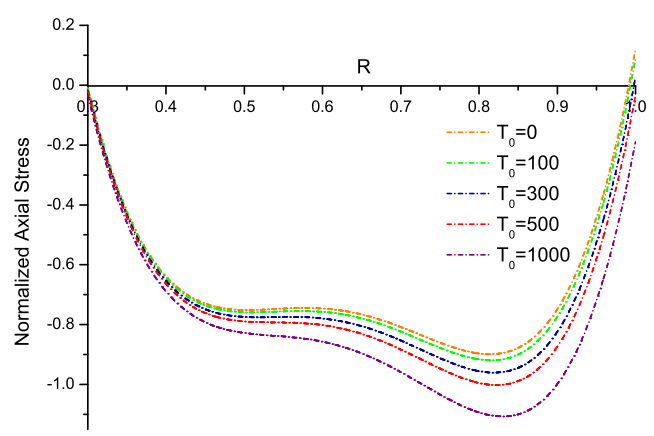

Figure 8: Normalized axial Stress distribution in FGM hollow cylinder with different values of $T_{0}$

Figures 6-8, represent thermoelastic characteristics in FGM hollow cylinder for $T_{0}=0^{\circ} \mathrm{C}, T_{0}=100^{\circ} \mathrm{C}, T_{0}=$ $300^{\circ} \mathrm{C}, T_{0}=500^{\circ} \mathrm{C}, T_{0}=1000^{\circ} \mathrm{C}$. It is observed from figures, that there is an inverse relation between normalized stresses and $T_{0}$ i.e. as the value of $T_{0}$ increases the values of normalized stress increase, otherwise the behaviour of curves is the same in nature as for different values of $T_{0}$. The maximum values of stress are attained for minimum value of $T_{0}$ i.e. $T_{0}=0$.

\section{CASE IV: For different values of Poisson's ratio:}

In this section, the effect of Poisson's ratio on normalized radial, circumferential and axial stress is studied by taking differnt values of Poisson's ratio.

It is observed from Figure 9, normalized radial stress is tensile in the range when $0.7 \leq v \leq 1$, whereas compressive for remaining range. It is converging to zero at

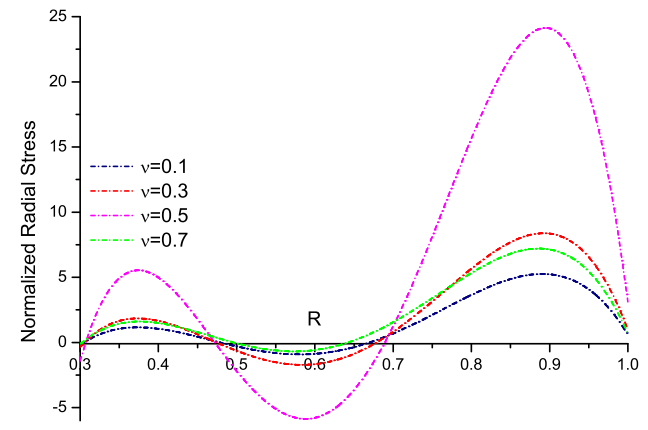

Figure 9: Variation of normalized radial stress with Poisson's ratio

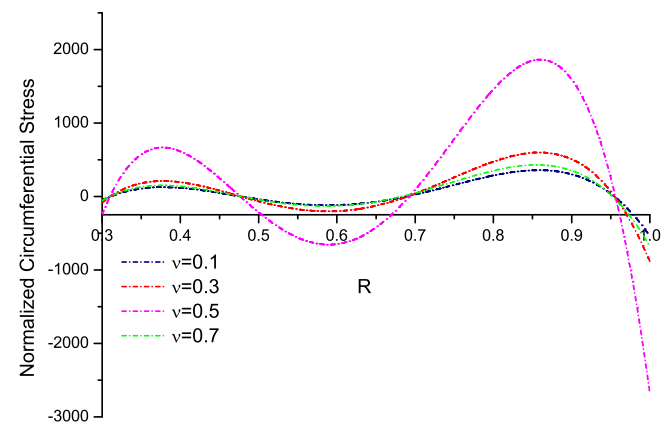

Figure 10: Variation of normalized circumferential stress with Poisson's ratio

the central region. The maximum value of normalized radial stress is obtained when Poisson's ratio is 0.5. Figure 10 , shown variation of normalized circumferential stress in radial direction for different values of Poisson's ratio. The normalized circumferential stress is elastic in nature for starting and ending values of dimensionless radial coordinate. The maximum variation exists in nature of curve when the value of Poisson's ratio is taken as 0.5. In tensile region the behaviour of Normalized curve is firstly increasing in nature, after mid of region then the behaviour of curve decreasing in nature. Figure 11, represents variation of normalized axial stress for different values Poisson's ratio. The magnitude of axial stress is high for outer surface and confining in nature for middle values of $R$. The behaviour of axial stress curve is compatible for different of Poisson's ratio.

\section{Material Properties:}

Figures 12-16 represent a material properties corresponding to different values of $\beta$. From Figure 12, it is ob- 


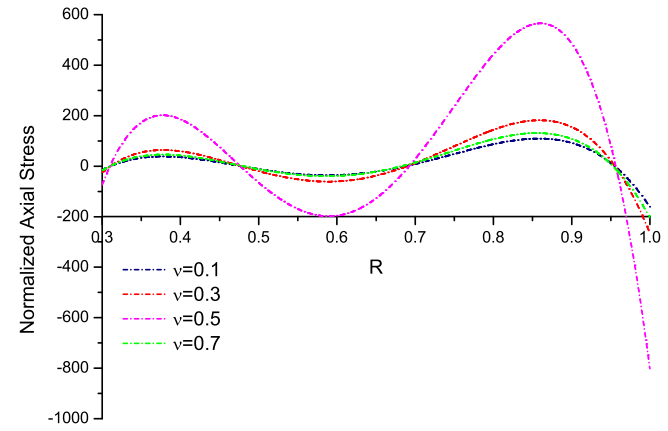

Figure 11: Variation of normalized axial stress with Poisson's ratio

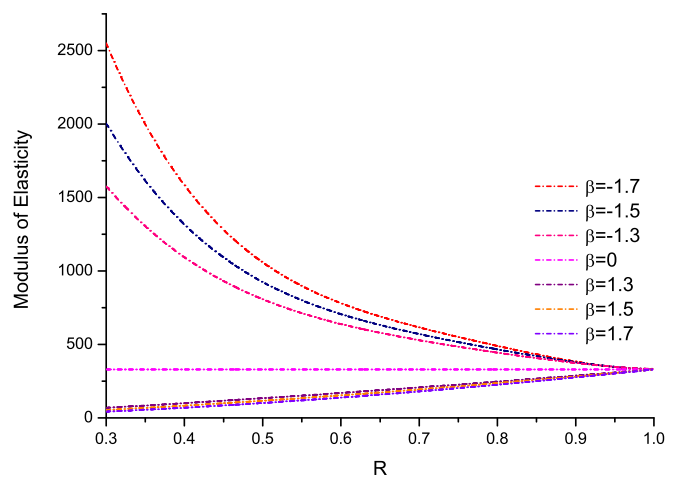

Figure 12: Distribution of modulus of elasticity along radial direction for different values of $\beta$

served that modulus of elasticity decreases as the value of $\beta$ increases. The behaviour of modulus of elasticity curve same for different values of $\beta$. From Figure 13 it is cleared that maximum variation in thermal conductivity coefficient for values of $\beta$ exists when $0.3 \leq \beta \leq 0.6$. The behaviour of curve is same in nature for different values of $\beta$. Figure 14 , shown that the behaviour of thermal conductivity coefficient curve is different for negative and positive values of $\beta$. For negative values of $\beta$ the behaviour of curve is decreasing in nature on the other hand for positive values of $\beta$ it is increasing in nature. From Figure 15, It is observed that maximum value of normalized stress is obtained when $\beta=-1.7$ and minimum value for $\beta=1.7$. Figure 16, shown that the behaviour of normalized temperature is decreasing for all values of $\beta$.

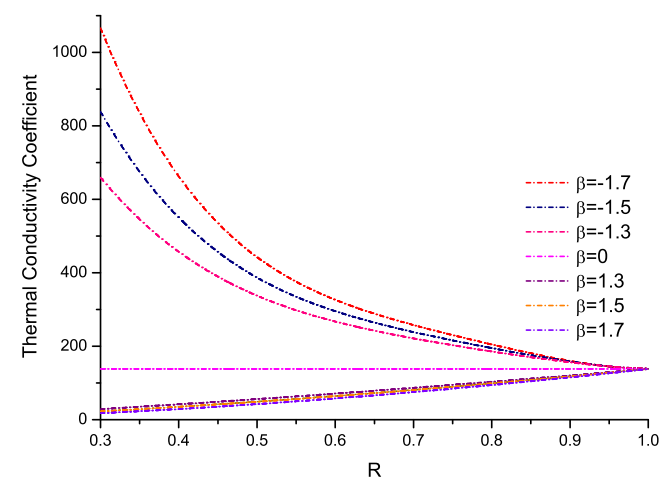

Figure 13: Distribution of thermal conductivity coefficient along radial direction for different values of $\beta$.

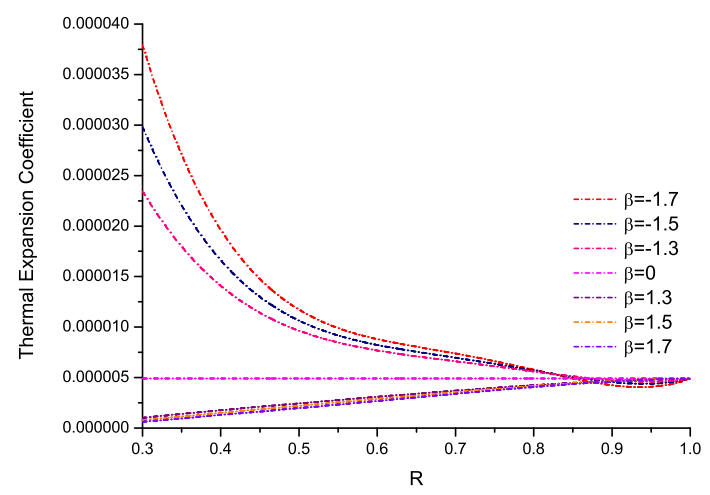

Figure 14: Distribution of thermal expansion coefficient along radial direction for different values of $\beta$

\section{Conclusion}

In the present paper, the thermoelastic analysis of functionally graded hollow cylinder with FEM under radially varing material properties is made. Normalized stress is obtained for different values of $T_{0}$. Further, the effect of Poisson's ration is investigated by changing the parameter $v$. The following conclusions could be drawn from the presented study:

- For anistropic hollow cylinder, maximum variation in stress exists for positive values of $\beta$ and minimum variation appears for negative values of $\beta$.

- For homogeneous cylinder the normalized radial stress is less than 0 , where the other two stress values are greater than 0 at the last values of $R$.

- By increasing the values of $T_{0}$, it is observed that value of normalized stress decreases. 


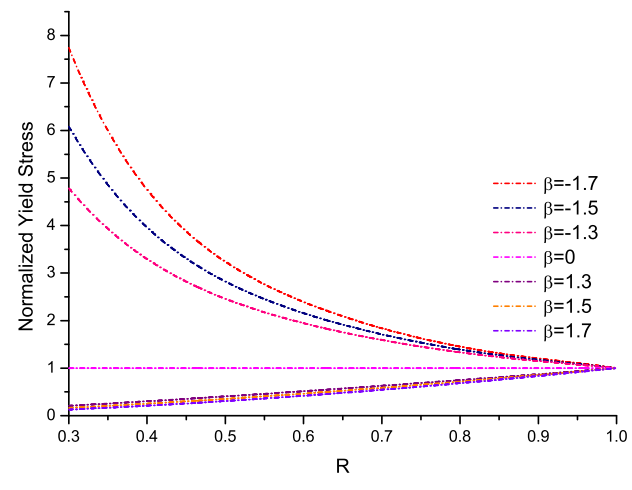

Figure 15: Distribution of normalized yield stress along radial direction for different values of $\beta$

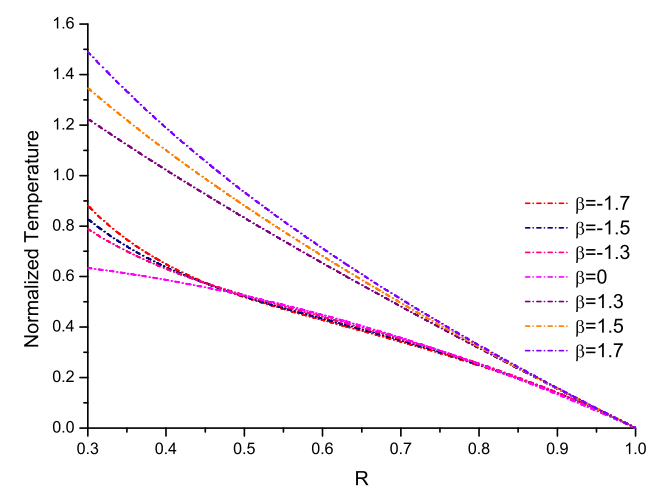

Figure 16: Distribution of normalized temperature along radial direction for different values of $\beta$

- Particular cases of Poisson's ratio study by assigning different values to $v$. The behaviour of normalized stress is highly fluctuating when $v=0.5$.

\section{References}

[1] Dai H.L., Rao Y.N., Jiang H.J., An analytical method for mangneto-thermoelastic analysis of functionally graded hollow cylinders, Appl. Math. Comput., 2018, 1467-1477.

[2] Abrinia K., Naee H., Sadeghi F., Djavanrood F., New Analysis for the FGM Thick cylinder under combined pressure and temperature loading Am. J. Appl. Sci., 2008, 5, 852-859.

[3] Evci C., Gulgec M., Functionally graded hollow cylinder under pressure and thermal loading: Effect of material parameters on stress and temperature distributions, Int. J. Eng. Sci., 2018, 92-108.

[4] Rahimi G.H., Nejad M. Zamani. 2008. Exact solutions for thermal stresses in rotating thick-walled cylinder of functionally graded material, J. Appl. Sci., 3267-3272.
[5] Sharma J.N., Sharma D., Kumar S., Analysis of stress and strains in rotating homogeneous thermoelastic circular disk by using finite element method, Int. J. Comput. Appl., 2011, 35.

[6] Sharma J.N., Sharma D., Kumar S., Vibration Analysis of a Rotating FGM Thermoelastic Axisymmetric Circular Disk Using FEM, Int. J. Comput. Method. Eng. Sci. Mech., 2013, 14.

[7] Naghdabadi R., Hosseini K.S.A., A Finite element formulation for analysis of functionally graded plates and shells, Arch. Appl. Mech., 2005, 74, 375-386.

[8] Go J. 2019. Mathematical approach to thermoelastic characteristics for functionally graded rotating circular disks. Appl. Math. Sci., 13: 533-545.

[9] Reddy J.N., An introduction to finite element method, 2005, McGraw Hill Education.

[10] Zienkiewicz O.C., Taylor R.L., The finite element method its basis and fundaments, 2005, Elsevier.

[11] Cook R.D., Malkus D.S., Pleasha M.E., Witt R.J., Concept and application of finite element method, 2007, Wiley.

[12] Hutton D.V., Fundamental of finite element analysis, 2003, McGraw Hill Education.

[13] Yadav O.M., Jiwari R., Finite element analysis and approximation of Burger's fisher equation. Numer. Meth. Part. D.E., 2017, 33, 1652-1677.

[14] Yadav O.M., Jiwari R., A finite element approach to capture turing patterns of autocatalytic brusselator model, J. Math. Chem., 2019, 57, 769-789.

[15] Yadav 0.M., Jiwari R., A finite element approach for analysis and computational modelling of coupled reaction diffusion models. Numer. Meth. Part. D.E., 2018, 35, 830-850.

[16] Sharma D., Kaur R., Sharma H., Analysis of thermoelastic characteristics of disk using linear properties of material. Malaya J. Mat., 2019, 7, 632-642.

[17] Sharma D., Kaur R., Finite element solution for stress and strain in FGM circular disk, AIP Conf. Proc., 2019, 2142.

[18] Farhan A.M., Abd-Alla A.M., Khder A.M., Solution of a problem of thermal stresses in a non-homogeneeous thermoelastic infinite medium of isotropic material by finite difference method, J. Ocean Eng. Sci., 2019, 4, 256-262.

[19] Habib E.S., El-Hadek M.A., El-Megharbel A., Stress analysis for cylinder made of FGM and subjected to thermo-mechanical loadings, Metals, 2019, 9.

[20] Vaziri S.A., Ghannad M., Beg O.A., Exact thermoelastic analysis of a thick cylindrical functionally graded material shell under unsteady heating using first order shear deformation theory, Heat Tran. Asian Res., 2019, 45, 1737-1760.

[21] Bayat M., Saleem M., Sahari B.B., Hamouda A.M.S., Mahdi E., Mechanical and thermal stresses in functionally graded rotating disk with variable thickness due radially symmetry loads, Int. J. Pres. Ves. Pip., 2009, 86, 357-372.

[22] Liew K.M., Kitipornchai S., Zhang X.Z., Lim C.W., Analysis of the thermal stress behaviour of functionally graded hollow circular cylinders, Int. J. Solids Struct., 2003, 40, 2355-2380.

[23] Jabbari M., Bahtui A., Eslami M.R., Axisymmetric mechanical and thermal stress in thick short length FGM cylinders, Int. J. Pres. Ves. Pip., 2009, 86, 296-306.

[24] Parveen G.N., Chin C.D., Reddy J.N., Thermoelastic Analysis of functionally graded ceramic metal cylinder, J. Eng. Mech., 1999, 125,1259-1267.

[25] Jabbari M., Sohrabpou S., Eslami M.R., Mechanical and Thermal stresses in a functionally graded hollow cylinder due to 
radially symmetric loads, Int. J. Pres. Ves. Pip., 2002, 79, 493497.

[26] Nejad M.Z., Abedi M., Lotfian M.H., Ghannd M., Exact and numerical elastic analysis for the FGM thick-walled cylindrical pressure vessels with exponentially-varing properties, Arch. Metall. Mater., 2016, 61,1649-1654.

[27] Eslami M.R., Babaei M.H., Poultangari R., Thermal and mechanical stresses in a functionally graded thick sphere, Int. J. Pres. Ves. Pip., 2005, 82, 522-527.

[28] Peng X.L., Li X.F., Thermoelastic analysis of cylindrical vessel of functionally graded materials, Int. J. Pres. Ves. Pip., 2010, 87, 203-210.

[29] Yeo W.H., Purbolaksono J., Aliabadi M.H., Ramesh S., Liew H.L., Exact solutions for stresses/displacements in a multilayered hollow cylinder under thermo-mechanical loading, Int. J. Pres. Ves. Pip.,2017, 151.

[30] Atli A.A., Lak D., Analytic solution of effect of electric field on elasto-plastic response of functionally graded piezoelectric hollow cylinder, Int. J. Pres. Ves. Pip., 2017, 155, 1-14.

[31] Tutuncu N., Stresses in thick walled FGM cylinders with exponentially varing properties, Eng. Struct., 2007, 29, 2032-2035.
[32] Wang H.M., An effective approach for transient thermal analysis in a functionally graded hollow cylinder, Int. J. Heat Mass Transf., 2013, 67, 499-505.

[33] Tanvir A.N.M., Islam M.D., Ahmed F., Analysis of Thermoelastic characteristics in a thick walled FGM cylinder, AIP Conf. Proc., 2017, 1919.

[34] Zheng Y., Bahaloo H., Mousanezhad D., Mahdi E., Vaziri A., Nayeb-Hashemi H., Stress Analysis in functionally graded rotating disk with non-uniform thickness and variable angular velocity, Int. J. Mech. Sci., 2016, 119.

[35] Zenkour A.M., Exact solution of thermal stress problem of an inhomogeneous hydrothermal piezoelectric hollow cylinder, Appl. Math. Model., 2014, 38, 6133-6143.

[36] Manthena V.R., Lamba N.K., Kedar G.D., Mathematical modeling of thermoelastic state of a thick hollow cylinder with non homogeneous material properties, J. Solid Mech., 2018, 10, 142-156.

[37] Saddalfar M., Stress redistribution analysis of piezomagnetic rotating thick-walled cylinder with temperature and moisture dependent material properties, J. Appl. Comput. Mech., 2019, 6. 